\title{
The effect of burst duration, interstimulus onset interval, and loudspeaker arrangement on auditory apparent motion in the free field ${ }^{a)}$
}

\author{
Thomas Z Strybel \\ California State University Long Beach, Department of Psychology, Long Beach, California 90840 \\ Wayne Neale \\ Virginia Polytechnic Institute and State University, Department of Industrial and Systems Engineering, \\ Blacksburg, Virginia 24061 \\ (Received 9 November 1992; accepted for publication 12 July 1994)
}

\begin{abstract}
The illusion of auditory apparent motion (AAM) was examined in order to determine the burst durations and interstimulus onset intervals (ISOIs) at which AAM is heard when spatial information regarding source location was varied. In the first experiment AAM was examined in the free field under monaural and binaural listening conditions. AAM was heard at the same burst duration-ISOI combinations for both listening conditions, but the location of the lead source could be determined only under binaural listening. In the second experiment AAM was measured with two and three sound sources. The number of sources did not affect the burst duration-ISOI combinations that produced AAM, but did affect the determination of the location of the lead source. In the third experiment AAM was tested when the sources were located in the median plane. The sources were located either at $0^{\circ}$ and $180^{\circ}$ azimuth, or both at $0^{\circ}$ azimuth, one in the horizontal plane and one $20^{\circ}$ above. The location of the speakers did not affect the timing requirements for the perception of AAM, only the timing requirements for the detection of the lead source. In the fourth experiment, AAM was measured when the vertical separation between the sources was either $2.5^{\circ}$ or $20^{\circ}$. AAM was heard at both separations, even though $2.5^{\circ}$ is less than the vertical MAA. In each of these experiments only burst duration and ISOI determined whether motion was heard. Localization cues were important only for the determination of the direction of motion.
\end{abstract}

PACS numbers: 43.66.Mk, 43.66.Pn, 43.66.Qp [HSC]

\section{INTRODUCTION}

For the past 20 years the primary method of investigating auditory motion perception has been the determination of the minimum audible movement angle (MAMA), a measure of dynamic acuity. The MAMA is defined as either the minimum extent of travel required for identification of the direction of sound source movement (e.g., left or right) or the minimum extent of travel that allows accurate discrimination between a moving and stationary stimulus (e.g., Perrott and Musicant, 1977; Grantham, 1986). MAMAs have been measured under a variety of conditions. The results of these experiments indicate that factors affecting performance on static acuity tasks also affect performance on dynamic acuity tasks. For example, the MAMA is smallest $\left(1-2^{\circ}\right)$ when the stimulus consists of broadband noise sources located directly in front of the listener (e.g., Strybel et al., 1992; Chandler and Grantham, 1992; Perrott and Tucker, 1988; Grantham, 1986; for a review see Middlebrooks and Green, 1991). These findings are similar to those of Mills (1958) on the minimum audible angle (MAA), a measure of static acuity. One factor that influences the MAMA but by definition cannot affect the MAA is the velocity of the moving sound. Saberi and Perrott (1990) determined that the relationship

\footnotetext{
a) Portions of this paper were presented at the 33rd Annual Meeting of the Psychonomic Society, St. Louis, MO, and the 125th Meeting of the Acoustical Society of America, Denver, CO.
}

between the MAMA and velocity is U-shaped, with the lowest MAMAs obtained at a velocity of $2-10 \%$.

The results of research on the MAMA have been explained by a snapshot mechanism. This hypothesis was first stated in Grantham (1986) and was recently reviewed in Middlebrooks and Green (1991). According to the snapshot hypothesis, the auditory system detects motion by determining the location of a moving sound at two points in time. If a change in the location of the sound is detected, motion is heard. According to this hypothesis, auditory motion is not processed by specialized auditory motion detectors. The snapshot hypothesis is consistent with the research on MAMAs as it predicts that factors affecting static localization would also influence the detection of moving sounds.

Grantham (1986) added that a minimum integration period of $150-300 \mathrm{~ms}$ is required for optimal performance on MAMA tasks. When sounds travel for less than this time period, the MAMA is elevated, because spatial resolution becomes poor as the duration of a moving target decreases below $150 \mathrm{~ms}$. This was confirmed by the results of Perrott and Pacheco (1989), who showed that the MAA was optimal when the temporal separation between the static sounds exceeded 100-150 ms. The minimum integration period could explain why the MAMA increases with velocity for velocities greater than $2 \%$. At velocities lower than $2 \%$, where the MAMA is inversely related to velocity, Middlebrooks and Green (1991) suggest that the elevation in MAMAs is caused 
by memory limitations. Thus the snapshot hypothesis can account for most of the data on the MAMA.

The MAMA has been measured both for real moving sounds and for simulations of sound movement. Simulations of movement are created by replicating one or more of the dynamic features of a sound in motion. Some simulations replicate dynamic interaural intensity and time differences (e.g., Grantham, 1986). Others replicate only dynamic interaural time differences (e.g., Altman and Viskov, 1977). Generally speaking, MAMAs obtained with simulated movement are similar to those obtained with real movement (Grantham 1986; Perrott and Tucker, 1988). These simulation techniques are important because they are relatively easy to create and they can be used to determine the essential features of a moving stimulus that produce the perception of auditory motion. For example, the snapshot hypothesis predicts that motion can be heard with a minimum of two looks at a moving sound stimulus. This prediction could be tested with a simulation consisting of only two different values of interaural time or intensity differences. A simulation of this type was reported by Viskov (1975), who used a dichotic-click technique that replicated the dynamical interaural time differences of a moving sound source. He determined that motion could be heard when only two clicks were presented.

Similarly, the snapshot hypothesis predicts that the simplest simulation technique in the free field is two sound bursts separated in time and space. A simulation of this type has been created, and is known as auditory apparent motion (AAM). AAM is an important method for investigating auditory motion not only because it represents the minimum conditions for motion, but also because apparent motion can be produced in the visual and tactual modalities. This suggests that similarities between the senses exist at some level in the processing of motion. In vision there are probably more published reports of the illusion of visual apparent motion than of real visual motion (Kolers, 1972). This is probably due to the assumption that visual apparent motion contains only the essential stimulus features that can produce the perception of motion (e.g., Newsome et al., 1986). In fact, many recent theories of visual motion perception are based almost exclusively on this illusion (for a review see Mack, 1986).

\section{A. Auditory apparent motion}

AAM is heard when two sounds presented in sequence are appropriately timed. The important timing parameters are the interstimulus onset interval (ISOI), or the time between the onset of the lead and lag stimulus, and the duration of each stimulus, known as burst duration. When the ISOI and burst duration are appropriate, the sound is heard to move from the location of the lead stimulus to the location of the lag stimulus. The auditory illusion was first reported by Burtt (1917), and Briggs and Perrott (1972) were the first modern investigators of the illusion. Briggs and Perrott investigated dichotic AAM and determined that the perceptual experience of the sound sequence depended on ISOI and burst duration. For ISOIs around $0 \mathrm{~ms}$, listeners reported hearing either one or two stationary sound images. As the ISOI increased, first a single sound image was heard to move between the ears and then an interrupted moving sound was heard. The former type of movement was called continuous motion and the latter broken motion. At the highest ISOIs tested, two nonmoving sounds were heard in succession. The exact values of ISOI that produced each perception depended on burst duration.

Subsequent research on AAM has examined the role of temporal and spatial factors in the illusion. In general, this research has shown that timing factors (burst duration and ISOI) are more important than spatial factors (e.g., binaural localization cues). Briggs and Perrott (1972) determined that the ISOI that produced AAM is a function of the duration of the stimuli. As duration increased, the ISOIs that produced the illusion also increased, but at a slower rate. In vision, a similar finding has been reported, and this relationship between burst duration and ISOI is known as Korte's second law (Kolers, 1972). Strybel et al. (1992) determined that a minimum burst duration between 10-50 ms was required for the perception of continuous motion. The optimal conditions for continuous movement in this experiment were a burst duration and ISOI of $50 \mathrm{~ms}$.

When the effects of spatial factors on AAM are examined, it appears that cues to the location of the sound sources do not affect whether motion is heard, but do affect whether the direction of the apparently moving stimulus can be determined. Strybel et al. (1989) reported that AAM can be heard under both monaural and binaural listening conditions for roughly the same range of ISOIs. The only difference between monaural and binaural AAM was that the direction of motion could not be identified in the monaural listening condition. Subjects (including the authors) in this experiment reported a clear sense of motion in both listening conditions. In the monaural condition, the extent of the path of the motion was shorter than the extent of the path in the binaural listening condition. Furthermore, in the monaural condition the detection of the direction of movement either could not be determined or the perceived direction was incorrect.

The range of timing values that produce AAM are also not affected by spatial separation. Strybel et al. (1990) determined that for separations between $6^{\circ}$ and $160^{\circ}$, AAM was heard at the same ISOI range. Strybel et al. (1992) extended the range of separations down to $2.5^{\circ}$, and determined that the minimum burst-duration requirement for continuous motion was not affected by the spatial separation between the sources. Although spatial separation had no effect on the ISOI range at which AAM was heard, spatial separation did affect whether the direction of motion could be detected. In vision, separation affects both the ISOIs at which apparent motion is heard and the ISOIs at which the direction of apparent motion can be detected (Strybel et al., 1990).

At the present time, the only explanation of AAM was proposed by Strybel et al. (1992). It was suggested in this report that the conditions favoring the perception of continuous motion were compatible with the minimum integration period of Grantham (1986). The optimal conditions for continuous motion in the Strybel et al. (1992) study were a stimulus sequence whose total duration (burst duration +ISOI) exceeded $100 \mathrm{~ms}$ and did not contain a silent gap. These conditions would explain the minimum burst duration 
requirement for continuous motion, because it is not possible to create these optimal conditions with burst durations less than $50 \mathrm{~ms}$ unless three or more sources are used to produce the illusion. However, in Strybel et al. (1992) the total duration of the stimulus sequence was confounded with burst duration and ISOI, and AAM was not measured at burst durations greater than $50 \mathrm{~ms}$.

The following set of experiments was undertaken in order to examine further the effect of spatial information on the timing parameters that produce AAM, and to examine the minimum burst duration requirement in more detail. Four experiments were run. The first experiment examined the effect of burst duration on AAM under binaural and monaural listening conditions. The second experiment tested the minimum burst duration requirement for AAM when the illusion was produced with either two or three sound sources. The third experiment measured the minimum burst duration requirement for sources separated in the median plane. The final experiment determined whether AAM could be perceived when the separation between the sources was less than one MAA.

\section{EXPERIMENT 1: THE EFFECTS OF BURST DURATION AND ISOI ON AAM UNDER MONAURAL AND BINAURAL LISTENING CONDITIONS}

Strybel et al. (1989) determined that AAM could be heard under both monaural and binaural listening conditions for ISOIs between 20 and $130 \mathrm{~ms}$. Differences between listening conditions were obtained only when subjects reported the perceived direction of the apparently moving sound (leftright or vice versa). In the binaural listening condition, the direction of the moving source could be identified at levels exceeding $90 \%$ for ISOIs in excess of $10-20 \mathrm{~ms}$ at all separations tested $\left(10^{\circ}-160^{\circ}\right)$. In the monaural condition, direction of motion could be identified only at ISOIs greater than $300 \mathrm{~ms}$ when the separation between the sources was $40^{\circ}$, and at ISOIs greater than $130 \mathrm{~ms}$ when the spatial separation was $160^{\circ}$. For the $10^{\circ}$ separation, performance was at chance levels at all ISOIs. In effect, the location of the individual sounds could be determined under monaural listening conditions only when the ISOI exceeded $130 \mathrm{~ms}$, the maximum ISOI value at which the illusion of movement was heard. These findings were obtained with a single value of burst duration, $50 \mathrm{~ms}$. The present experiment therefore manipulated both burst duration and ISOI under monaural and binaural listening conditions using a stimulus that could not be accurately localized in the monaural listening condition.

\section{A. Method}

\section{Subjects}

Six subjects were tested (including one of the authors, WN), all with normal hearing. Three subjects had previous experience in auditory apparent motion studies and three subjects were naive listeners.

\section{Apparatus}

Testing was conducted in a $2.7 \times 3.7 \times 2.1-\mathrm{m}$ audiometric chamber (nonanechoic). Subjects were seated in the center of the room, with the head fixed by a chin rest. Two speakers were positioned one meter from the subject and were separated by $40^{\circ}$. In the binaural listening condition, the subject was oriented so that the speakers were located at $\pm 20^{\circ}$ azimuth (separated by $40^{\circ}$ and directly in front of the subject). In the monaural listening condition, the subject was positioned so that the speakers were located at 70 and $110^{\circ}$ azimuth (separated by $40^{\circ}$ and opposite the right ear). An Amiga 1000 computer was used to generate and present the stimulus, control all timing, and collect the response of the subject. The responses were made on a box that contained five three-position switches attached to the game port of the computer.

The monaural listening condition was created by inserting a Noise Filter (Ear Group) earplug into the left ear of the subject and placing a Safety Direct ear muff over the left ear. The plugs reported an attenuation of at least $37.4 \mathrm{~dB}$ for frequencies of $0.5 \mathrm{kHz}$ or greater and the ear muffs report an attenuation of at least $27.6 \mathrm{~dB}$ for frequencies of $0.5 \mathrm{kHz}$ or greater.

\section{Procedure}

The stimulus consisted of random noise with a band of $2-4 \mathrm{kHz}$ and rise time of less than $1 \mathrm{~ms}$. The signal level was set at $5 \mathrm{~dB}$ below a subject's two-ear-occluded thresholds. This level was clearly audible to the unoccluded ear and yet below threshold for the occluded ear. Each subject participated in eight trial blocks, presented in random order. A trial block consisted of a particular burst duration $(10,50$, 100 , and $300 \mathrm{~ms}$ ) and listening condition (monaural or binaural). Within a trial block, 12 ISOI's $(0,20,30,40,50,60$, $70,110,150,200,300$, and $500 \mathrm{~ms}$ ) were presented 32 times each in random order for a total of 384 trials. At each ISOI, half of the trials originated from the right or forward speaker and half originated from the left or rearward speaker.

Subjects were instructed to classify their perception of the stimulus sequence into one of five categories developed by Briggs and Perrott (1972): Single, a single sound was heard with no movement; simultaneity, two sounds were heard at the same time at two different locations, with no movement between them; continuous motion, a sound was heard at one side that moved continuously toward the other side; broken motion, a sound was heard to move from one side to the other, but the movement was interrupted or discontinuous; and succession, two sounds were heard, one at one location then another at a different location, with no movement between them. Subjects were also instructed to indicate whether the lead sound originated from the left or right in the binaural listening condition and from the fore or aft in the monaural condition. This task required a judgment of temporal order for the stimuli that produced no motion, and a judgment of the direction of motion when motion was perceived. The category was indicated by selecting the appropriate switch, and the location of the lead stimulus by throwing it in the proper direction. Subjects initiated a trial block by depressing any switch on the response box. After a response was made to a stimulus sequence, the subsequent pair of stimuli followed $500 \mathrm{~ms}$ later. 

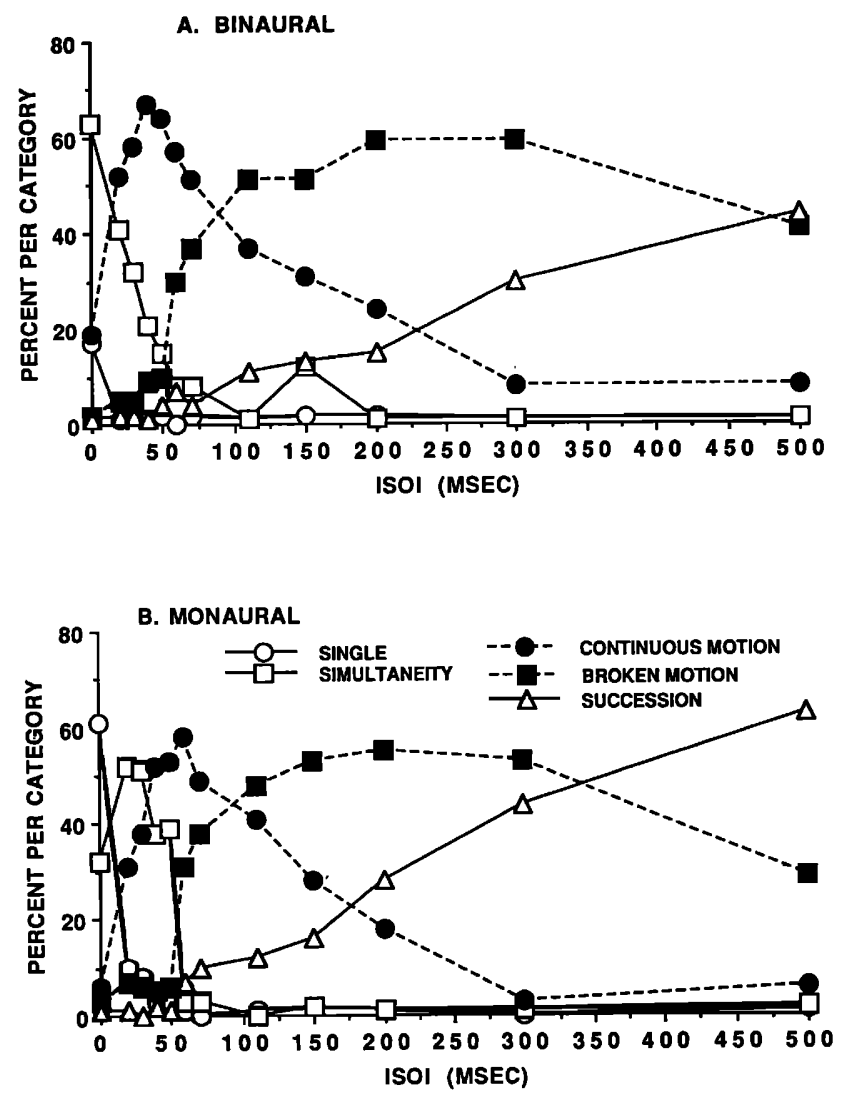

FIG. 1. The distribution of responses in each category as a function of ISOI under monaural and binaural listening conditions at a burst duration of 50 ms [(a) binaural listening; (b) monaural listening].

\section{B. Results and discussion}

The mean percentage of responses in each response category was determined as a function of ISOI, burst duration and listening condition. Figure 1 shows how the percentage of responses in each category were distributed across ISOI for a burst duration of $50 \mathrm{~ms}$. At the lowest ISOIs (0 and 20 $\mathrm{ms}$ ) the response category used most often was either single or simultaneity. In the binaural condition [Fig. 1(a)] only the simultaneity category was used, but in the monaural condition [Fig. 1(b)] single was dominant at $0 \mathrm{~ms}$ and simultaneity at $20 \mathrm{~ms}$. For ISOIs greater than $30 \mathrm{~ms}$ there was little difference in the distributions of responses between listening conditions. The dominant category was continuous motion for ISOIs between 30 and $70 \mathrm{~ms}$, broken motion for ISOIs between 150 and $200 \mathrm{~ms}$ and succession for ISOIs in excess of $200 \mathrm{~ms}$. Similar shifts in response category with ISOI were observed at each duration and listening condition, although the exact values at which each category was dominant depended on burst duration. In this and the following experiments, the discussion will focus on the continuous motion category because of the assumption that this type of motion is most like real motion.

A repeated measures analysis of variance was performed on the percentage of continuous motion responses, which are presented in Fig. 2. Significant main effects of listening condition $[F(1,5)=33.49 ; p<0.002]$, and ISOI $[F(11,55)$ $=6.23 ; p<0.02]$ were obtained. Overall, more continuous motion was reported in the binaural listening condition. This can be seen in each panel of Fig. 2 with the exception of the 10-ms duration [Fig. 2(a)]. In addition, continuous motion was reported only at a restricted range of ISOIs in both listening conditions. Several significant interactions were also found.

A significant interaction of duration by ISOI was obtained $[F(33,165)=2.92 ; p<0.0001]$. The ISOIs that produced the highest percentage of continuous motion responses depended on burst duration. As burst duration in-
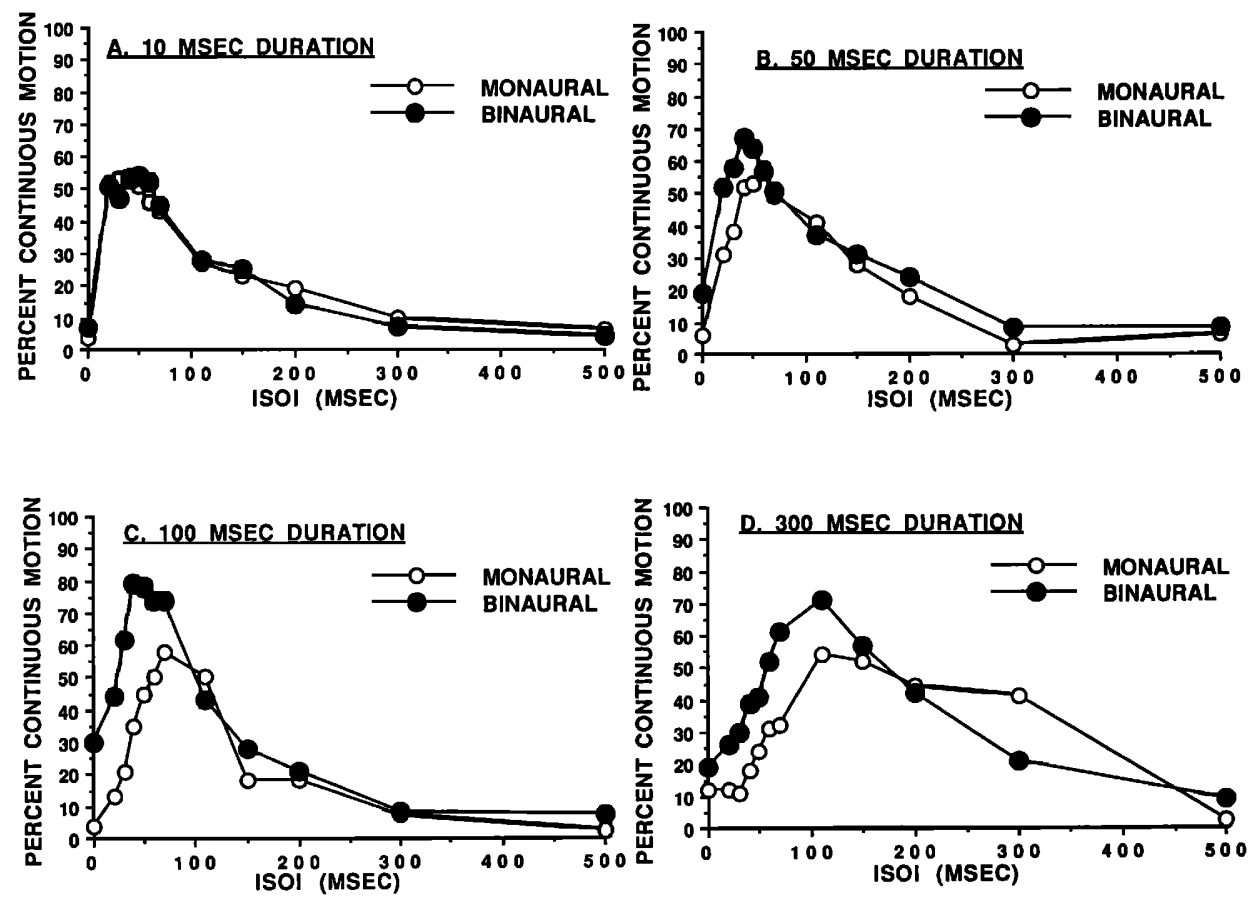

FIG. 2. The mean percentage of continuous motion responses as a function of ISOI under monaural and binaural listening conditions $[(a)$ burst duration $=10$ $\mathrm{ms}$; (b) burst duration $=50 \mathrm{~ms}$; (c) burst duration=100 ms; (d) burst duration=300 $\mathrm{ms}]$. 
TABLE I. Interstimulus onset interval (ISOI) threshold for the continuous motion as a function of burst duration and speaker arrangement.

\begin{tabular}{|c|c|c|c|c|}
\hline \multirow[b]{2}{*}{ Burst duration } & \multicolumn{4}{|c|}{ Threshold in ms } \\
\hline & Upper & Lower & Upper & Lower \\
\hline & \multicolumn{4}{|c|}{ Listening condition } \\
\hline & \multicolumn{2}{|c|}{ Monaural } & \multicolumn{2}{|c|}{ Binaural } \\
\hline 10 & 18 & 55 & 20 & 58 \\
\hline 50 & 39 & 68 & 19 & 72 \\
\hline 100 & 60 & 90 & 23 & 100 \\
\hline \multirow[t]{3}{*}{300} & 103 & 163 & 58 & 106 \\
\hline & \multicolumn{4}{|c|}{ Number of speakers } \\
\hline & \multicolumn{2}{|c|}{ Two } & \multicolumn{2}{|c|}{ Three } \\
\hline 5 & $\cdots$ & ... & $\cdots$ & $\cdots$ \\
\hline 10 & $\cdots$ & $\cdots$ & $\cdots$ & $\cdots$ \\
\hline 25 & 18 & 35 & 22 & 30 \\
\hline \multirow[t]{3}{*}{50} & 10 & 53 & 17 & 59 \\
\hline & \multicolumn{4}{|c|}{ Speaker arrangement } \\
\hline & \multicolumn{2}{|c|}{ Vertical } & \multicolumn{2}{|c|}{ Front-Back } \\
\hline 10 & $\cdots$ & $\cdots$ & $\ldots$ & $\cdots$ \\
\hline 25 & 22 & 24 & 24 & 26 \\
\hline \multirow[t]{3}{*}{50} & 20 & 50 & 21 & 56 \\
\hline & \multicolumn{4}{|c|}{ Vertical separation } \\
\hline & \multicolumn{2}{|c|}{$2.5^{\circ}$} & \multicolumn{2}{|c|}{$20^{\circ}$} \\
\hline 50 & 33 & 52 & 22 & 55 \\
\hline 100 & 10 & $\mathbf{a}$ & 9 & $\mathbf{a}$ \\
\hline
\end{tabular}

${ }^{a}$ ISOI range insufficient for upper threshold at burst duration of $100 \mathrm{~ms}$.

creased the peaks of the continuous motion distributions were located at higher ISOIs. Significant interactions of listening condition by ISOI, $[F(11,55)=2.96 ; p<0.003]$ and listening condition by duration by ISOI $[F(33,165)$ $=1.63 ; p<0.02]$ were also obtained. However, the magnitude of the main effect of listening condition and the two interactions with listening condition were small. Taken together these three significant effects accounted for only $6 \%$ of the total variance (Kirk, 1982), whereas the main effect of ISOI accounted for $23 \%$ of the variance, and the duration by ISOI interaction $12 \%$.

Table I presents the upper and lower ISOI threshold of continuous motion at each duration and listening condition. The upper and lower thresholds were defined as the lowest and highest ISOI values that produced at least $50 \%$ continuous motion reports. For both monaural and binaural listening conditions, as the duration increases, the ISOI range also increases. Two differences between listening conditions were observed in Table I. In the monaural listening condition, both the lower and upper ISOI limits increase with burst duration. In the binaural listening condition, only the upper ISOI increases for burst durations of $10-100 \mathrm{~ms}$. As a result, the range of ISOIs that produce continuous motion are larger in the binaural listening condition.

These results shown in Fig. 2 and Table I indicate that the perception of continuous motion is determined by the critical timing values in AAM, and these timing values are only minimally affected by listening conditions. ${ }^{1}$ The results in the binaural listening condition are consistent with the previous research of Strybel et al. (1992) and Briggs and Perrott (1972). Strybel et al. showed that a minimum duration of $10-50 \mathrm{~ms}$ was required for reliable continuous mo-

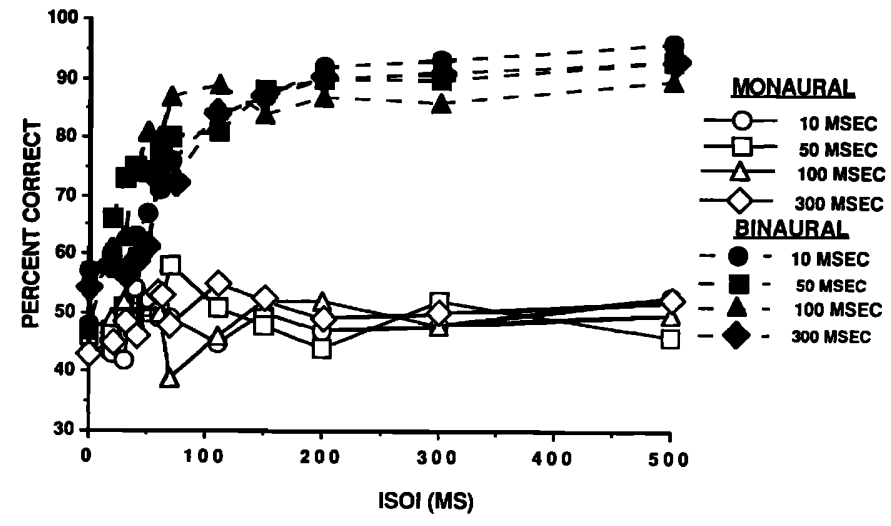

FIG. 3. The mean percentage of correct judgments of the direction of the lead source as a function of ISOI at all burst durations under monaural and binaural listening conditions.

tion reports, and that no silent gap should be produced in the stimulus sequence. As shown in Fig. 2, at the 10-ms duration the continuous motion distribution peaked at roughly $50 \%$, and at $50 \mathrm{~ms}$, the distribution peaked at roughly $70 \%$. This suggests that the minimum duration for continuous motion is roughly $10 \mathrm{~ms}$. In the monaural listening condition, a minimum duration requirement is more difficult to determine, as the peaks of these functions are between $50 \%-60 \%$ at all burst durations. Therefore, although the timing requirements are similar, monaural AAM might represent some minimum threshold condition for the perception of motion in the auditory modality at all burst durations.

The requirement of no silent gap does not predict when continuous motion will be heard at the longer burst durations. According to Strybel et al. (1992), if the minimum burst duration is exceeded and no silent gap is produced in the stimulus sequence, continuous motion should be heard. At a burst duration of $300 \mathrm{~ms}$, these conditions are met at all ISOIs less than $300 \mathrm{~ms}$. However, in Table I at $300 \mathrm{~ms}$, continuous motion is heard reliably only for ISOIs between 58 and $106 \mathrm{~ms}$. Broken motion was reported most often at ISOIs between 150 and $300 \mathrm{~ms}$, even though no silent gap was present. It appears that the conditions of a minimum total duration of $100 \mathrm{~ms}$ and no silent gap are necessary, but not sufficient conditions for continuous motion.

Similar findings at long burst durations were obtained by Briggs and Perrott (1972). These authors also determined that the effect of burst duration on the ISOIs that produced AAM can be described by Korte's second law of visual apparent motion (Kolers, 1972). This law states that as the burst duration increases, the ISOI that produces continuous motion also increases, but at a slower rate. Our results suggest that when the minimum burst duration is exceeded, Korte's second law is the best description of the durationISOI combinations that produce AAM, under binaural and monaural listening conditions.

The detection of the direction of AAM appears to be controlled by a different mechanism. Figure 3 presents the percentage of trials on which the location of the lead stimulus was correctly identified. It is evident from this figure that direction of motion could be determined only under binaural 
listening conditions. In the monaural listening condition, direction judgments were at chance levels for all durations and ISOIs. Using a repeated measures analysis of variance, significant effects of listening condition $[F(1,5)=26.4$; $p<0.004]$, ISOI $[F(11,55)=14.51 ; p<0.001]$ and condition by ISOI $[F(11,55)=9.17 ; p<0.001]$ were obtained. The main effect of listening condition accounted for $40 \%$ of the total variance, and the listening-condition by ISOI interaction 9\%. Poor performance on the direction of motion task was most likely caused by the spectrum of the stimulus which was limited to frequencies below $4 \mathrm{kHz}$. Monaural shape cues are effective only at frequencies above $8 \mathrm{kHz}^{2}$

In this experiment, only the temporal factors affected performance on the motion task, but temporal and spatial factors affected performance on the direction of motion task. This result is consistent with previous research on auditory AAM (e.g., Strybel et al., 1989). In addition, discrepancies between the detection of illusory motion and its displacement have been reported for visual illusions of motion. For example, after viewing a rotating spiral for some time period, stationary objects appear to contract or expand, but the perceived size of the visual object remains the same. As Gregory (1972) points out, laws describing the physical motion of objects may not pertain to illusory motion.

\section{EXPERIMENT 2: THE EFFECT OF BURST DURATION ON AAM WITH TWO AND THREE SOUND SOURCES}

In the previous experiment only the temporal variables of burst duration and ISOI determined when motion was heard. The following experiment investigated whether AAM is determined more by a minimum burst duration or by the total duration of the stimulus sequence. Total duration is equal to the sum of burst duration and ISOI when two speakers are used to produce the illusion.

If total duration is the best predictor of the illusion, then AAM could be heard with smaller burst durations when additional sound sources were used to create the illusion. For example, in Strybel et al. (1992) the optimal timing conditions for continuous motion was a burst duration and ISOI of $50 \mathrm{~ms}$, or total duration of $100 \mathrm{~ms}$. A total movement duration of $100 \mathrm{~ms}$ could also be produced with a burst duration and ISOI of $33.3 \mathrm{~ms}$ if three sources were used to produce the illusion (i.e., one at $-10^{\circ}, 0^{\circ}$ and $+10^{\circ}$ ). The potential importance of total movement duration was suggested by the report of Saberi and Perrott (1990) on the MAMA. These authors simulated motion using a burst durations of $1.5 \mathrm{~ms}$, well below the minimum duration requirement of $10 \mathrm{~ms}$, reported in Experiment 1 and in Strybel et al. (1992). However, Saberi and Perrott used an array of 15 speakers to simulate motion. It is possible that the increase in the number of speakers may have reduced the minimum burst duration requirement for the simulation of movement.

The following experiment was therefore performed to determine the effect of burst duration and ISOI on AAM produced with either two or three sound sources. We predicted that with three sound sources, motion would be heard at lower burst durations than with two sound sources.

\section{A. Method}

\section{Subjects}

Five subjects with normal hearing were tested in this experiment, three males and two females. All had participated in previous pilot research on AAM, but none had participated in experiment one.

\section{Apparatus}

High-pass noise $(2-8 \mathrm{kHz})$ was produced by three Quatech WSB-A12M 12-Bit Waveform Synthesizers and WSB100 Digital Pattern Generator Adapters mounted in the expansion slots of a IBM PC/AT compatible computer. The output of each board was amplified and led into one of three Realistic Minimus-7, two-way speakers. The level of each signal was set at $60 \mathrm{~dB}$ A-weighted with a rise time of less than $1 \mathrm{~ms}$. The speakers were located $1.1 \mathrm{~m}$ in front of the subject, at $-10^{\circ}, 0^{\circ}$, and $10^{\circ}$ azimuth. The AT computer controlled all timing, stimulus presentation, and response collection.

\section{Procedure}

In the two-speaker condition, the speaker at $0^{\circ}$ was not used. In the three speaker condition, all speakers were used. With this arrangement, the length of the path of the movement was the same in each condition $\left(20^{\circ}\right)$. Four burst durations were tested $(5,10,25$, and $50 \mathrm{~ms})$, with two and three speakers, for a total of eight trial blocks per subject. Within a trial block, 10 ISOIs $(2,4,8,10,15,25,35,45,55$, and 70 ms) were presented 30 times each, in random order. In the three-speaker condition, the ISOI represented the time between contiguous sound events. For example, in this condition a duration of $50 \mathrm{~ms}$ and ISOI of $45 \mathrm{~ms}$ meant that a stimulus of $50 \mathrm{~ms}$ from the left (right) speaker was followed by a 50 -ms stimulus from the center speaker $45 \mathrm{~ms}$ after the onset of the first stimulus. A third 50-ms pulse from the right (left) speaker occurred $45 \mathrm{~ms}$ after the onset of the center stimulus. Subjects were instructed to listen to the stimulus sequence and classify their perception into one of the five categories described in the previous experiment. In addition, subjects were required to identify the location of the lead stimulus (left or right).

\section{B. Results and discussion}

The percentage of responses in each category was determined. For ISOIs less than $15 \mathrm{~ms}$, the dominant response at all durations and number of speakers was simultaneity. At longer ISOIs, the motion response categories were dominant, with the particular category a function of number of speakers, burst duration and ISOI. The succession category was never dominant because the longest ISOI tested was only 70 ms.

The mean percentage of continuous motion responses are presented in Fig. 4 as a function of ISOI, burst duration and number of speakers. This figure indicates that the ISOIs producing continuous motion were not affected by the number of speakers used to produce the illusion. Again, only burst duration determined the ISOI range for continuous motion. For durations of 5 and $10 \mathrm{~ms}$, presented in Fig. 4(a) and 

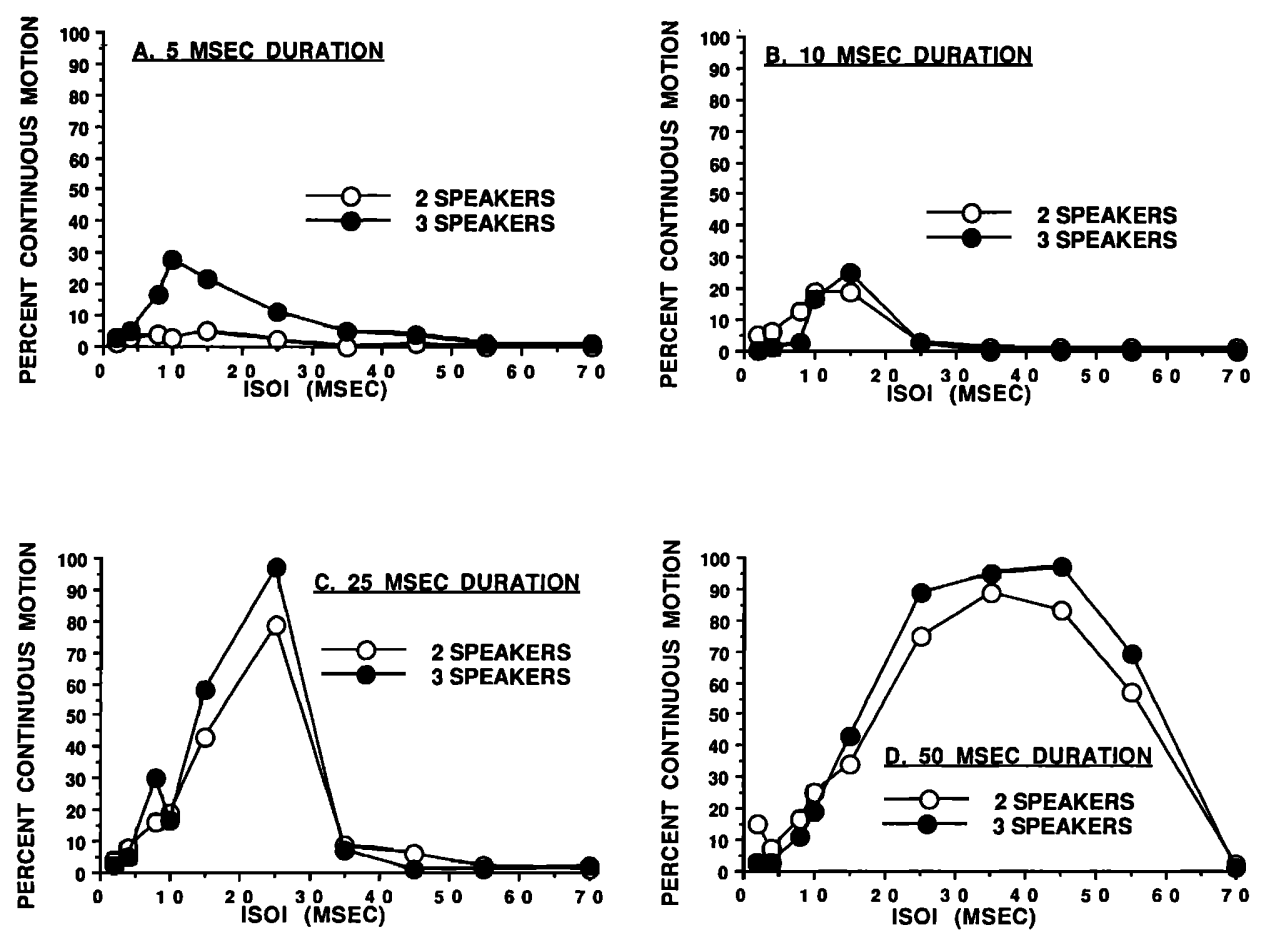

FIG. 4. The mean percentage of continuous motion responses as a function of ISOI for two and three sound sources [(a) burst duration=5 ms; (b) burst duration $=10 \mathrm{~ms}$; (c) burst duration $=25 \mathrm{~ms}$; (d) burst duration $=50 \mathrm{~ms}$ ].

(b), little continuous motion was reported. At the $25-\mathrm{ms}$ duration [Fig. 4(c)], the continuous motion distributions peak at an ISOI of $25 \mathrm{~ms}$. At the 50-ms duration [Fig. 4(c)], the peaks of the continuous motion distributions are broader and are shifted to the right. A repeated measures analysis of variance was performed on the percentage of continuous motion responses. No significant effect of the number of sources used to produce the illusion were found, nor were any significant interactions with the number of sources obtained. Only the main effects of duration $[F(3,12)=43.61$; $p<0.0001]$ and ISOI $[F(9,36)=38.27 ; p<0.0001]$ were significant. The only significant interaction was duration by ISOI $[F(27,108)=31.35 ; p<0.0001]$.

Table I presents the ISOI range which produced at least $50 \%$ continuous motion reports for 2 and 3 sound sources. This criterion was not met at burst durations of 5 and $10 \mathrm{~ms}$. At $25 \mathrm{~ms}$, continuous motion was heard at a narrow range of ISOIs, and at $50 \mathrm{~ms}$ the range is somewhat broader, due to the increase in the upper ISOI at which the criterion is met. There is no difference between numbers of speakers used in these ranges.

The results presented in Fig. 4 and Table I suggest that the number of sources do not affect the timing requirements for AAM. The minimum duration for continuous motion is between 10 and $25 \mathrm{~ms}$ for both two and three sources. In order to determine if total duration of the stimulus sequence is the appropriate predictor of AAM, the percentage of continuous motion responses are replotted in Fig. 5 as a function of the total duration of the stimulus sequence. The total duration of movement is the sum of burst duration and ISOI in the two-speaker condition, and the sum of burst duration and twice the ISOI in the three-speaker condition. If a total duration determines whether motion is heard, one would expect the peaks of these distributions at different burst durations and number of speakers to overlap. It is obvious from Fig. 5 that the continuous motion curves do not overlap. For continuous motion, the factors that produce the illusion are burst duration and ISOI only. ${ }^{3}$

The percentage of correct direction judgments are shown in Fig. 6 as a function of burst duration and ISOI [Fig. 6(a)], and total duration of movement [Fig. 6(b)]. A repeated measures analysis of variance on these data produced significant effects of number of sources $[F(1,4)=15.84 ; p<0.02]$, burst duration $[F(3,12)=13.42 ; p<0.0004]$, and ISOI $[F(9,36)=20.37 ; p<0.0001]$. As shown in Fig. 6(a), our listeners were more accurate with three speakers, and at longer burst durations and ISOIs. Significant interactions

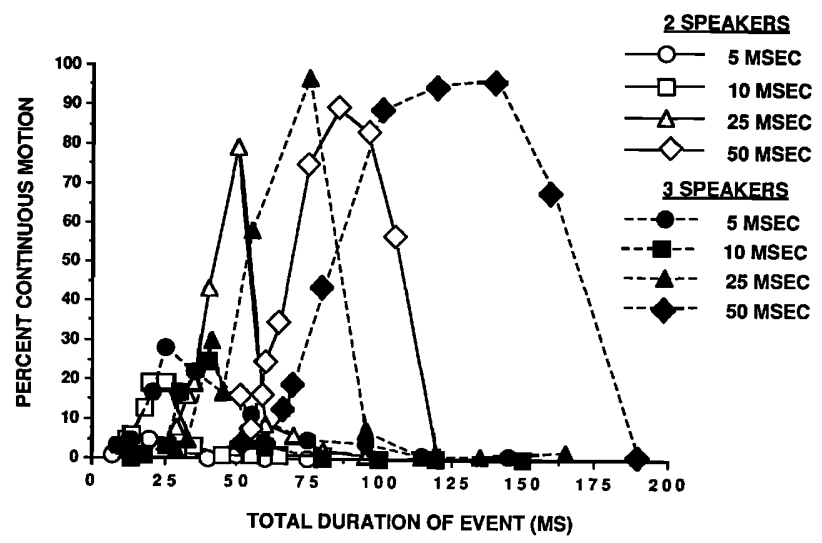

FIG. 5. The mean percentage of continuous motion responses as a function of the total duration of the event. Total duration was computed as the sum of burst duration and ISOI in the two-speaker condition, and the sum of burst duration and twice the ISOI in the three-speaker condition. 

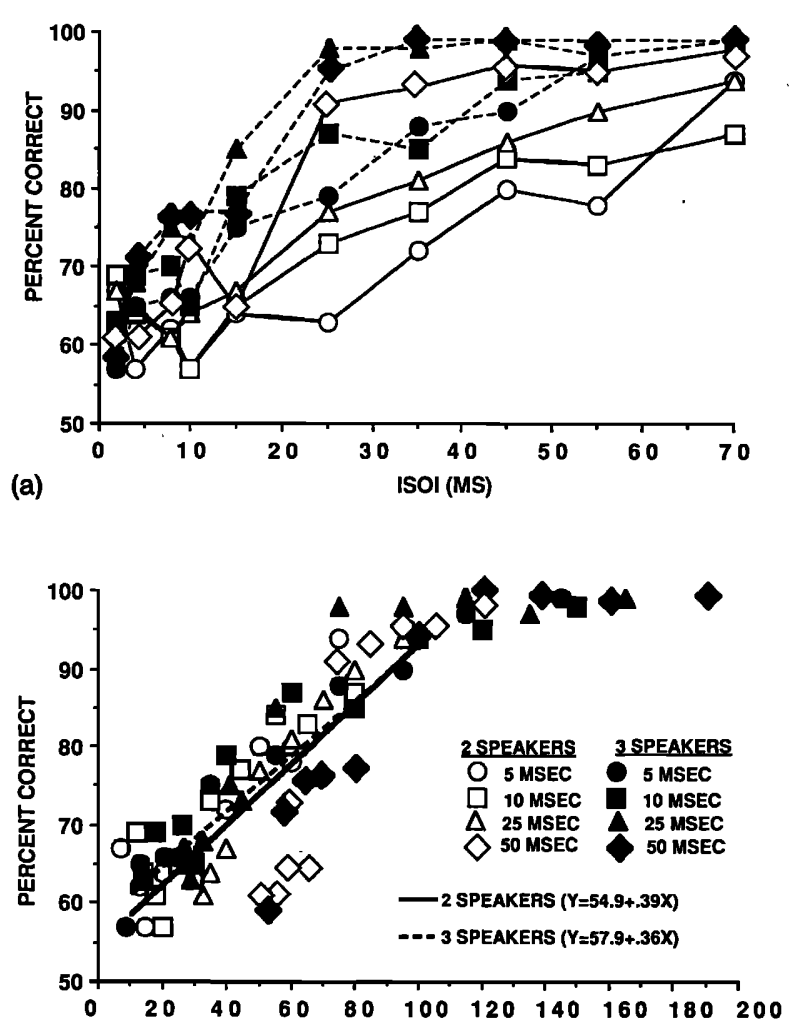

(b)

TOTAL DURATION OF EVENT (MS)

FIG. 6. The mean percentage of correct judgments of the direction of the lead source as a function of (a) ISOI and (b) total duration of the event for all burst durations and number of speakers tested.

were obtained between number of sources and ISOI $[F(9,36)=2.58 ; p<0.03]$ and burst duration and ISOI $[F(27,108)=1.74 ; p<0.03]$. Performance on the direction task was better for the three-speaker conditions, and for the longer burst durations.

These data are replotted in Fig. 6(b) as a function of total duration. This figure indicates that total duration of the stimulus sequence can predict whether or not the direction of the lead source was reliably detected. Separate least-squares curve-fits were calculated for two and three sources only for total movement durations less than $100 \mathrm{~ms}$ because the curves asymptote above this value. These functions [presented in Fig. 6(b)] have nearly identical slopes and intercepts, and each curve accounted for approximately $75 \%$ of the total variance. On the basis of these functions, the minimum total duration of movement required for the detection of the direction of movement is between 48 and $51 \mathrm{~ms}$, based on a performance criterion of $75 \%$ correct.

The results of experiment 2 indicate that total duration is not a reliable predictor of AAM, as suggested previously in Strybel et al. (1992). For continuous motion, the critical factors are burst duration and ISOI of each individual source. Furthermore, the results indicate again that motion is processed differently than the direction of motion, as performance on the direction task did depend on total duration of the sound sequence. In essence, subjects in this experiment used the duration and ISOI of contiguous events to determine whether the sound was moving, and compared the locations of the starting and ending points to determine the direction of motion. ${ }^{4}$

\section{EXPERIMENT 3: AAM IN THE MEDIAN PLANE}

The third experiment determined if AAM could be heard when the speakers were located in the median plane. We determined if the effect of burst duration in this experiment was similar to the effect noted in experiment one, using a stimulus (high-pass noise) that provided monaural shape cues about the location of each source.

\section{A. Method}

All of the subjects who served in experiment 2 were tested. Two speaker arrangements were used, vertical and front-back. In the vertical arrangement, two speakers were located at $0^{\circ}$ azimuth, one on the horizontal plane, and one $20^{\circ}$ above. In the front-back arrangement, two speakers were located in the horizontal plane, one speaker at $0^{\circ}$, and one at $180^{\circ}$. All speakers were located $1.1 \mathrm{~m}$ from the subject. The stimulus was the same as in experiment 2 . For each condition, three burst durations were tested $(10,25$, and $50 \mathrm{~ms})$ for a total of six trial blocks, presented in random order. Within each trial block, ten ISOIs were presented 30 times each in random order. The ISOIs used were the same as in experiment 2. Subjects were instructed to categorize their perception of the stimulus into one of the five categories defined previously. For judgments of location of the first occurring stimulus, subjects indicated "down" or "up" in the vertical arrangement and "front" or "back" in the front-back arrangement by throwing the appropriate switch in the indicated direction.

\section{B. Results and discussion}

The mean percentage of responses was determined for each ISOI, duration and speaker arrangement. As in the previous experiments, the percentage of continuous motion reports were analyzed with repeated measures ANOVAs. The data from experiment 2 in the two-speaker condition at equivalent durations were included in this analysis, providing three levels of speaker arrangement(vertical, front-back, and horizontal).

The percentage of continuous motion responses are presented in Fig. 7. It is evident from this figure that the arrangement of the sound sources had little effect on the ISOIburst duration combinations that produced continuous motion. Significant main effects of burst duration $[F(2,8)$ $=73.35 ; p<0.0001]$ and ISOI $[F(9,36)=16.7 ; p<0.0001]$ were obtained from the analysis of variance. As in experiments 1 and 2, more continuous motion was reported at the longer durations and at longer ISOIs. Significant interactions of speaker arrangement by ISOI $[F(18,72)=3.12$; $p<0.0003]$ and duration by ISOI $[F(18,72)=15.46$; $p<0.0001]$ were also found. However, the speaker arrangement by ISOI interaction accounted for only $2 \%$ of the total variance, while the duration by ISOI interaction accounted for $24 \%$ of the variance. 5

Table I presents the ISOI range that produced at least $50 \%$ continuous motion reports in all three speaker arrange- 

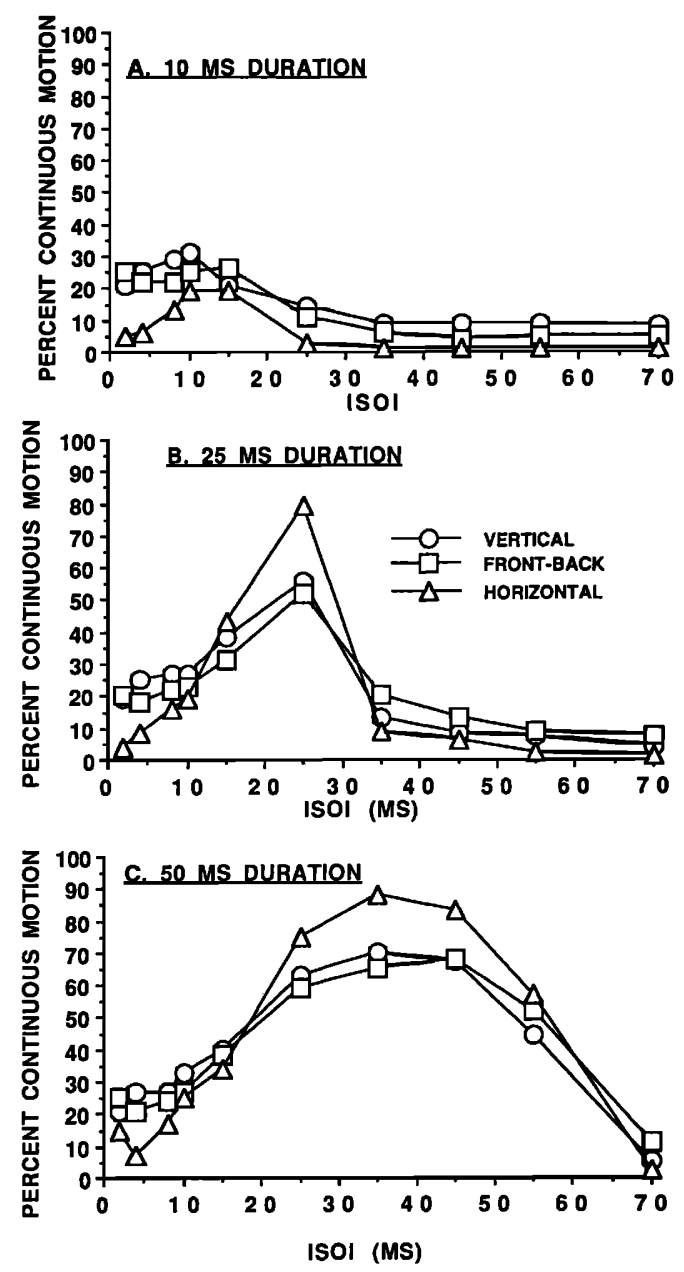

FIG. 7. The mean percentage of continuous motion responses as a function of ISOI and the arrangement of the speakers [(a) burst duration=10 ms; (b) burst duration $=25 \mathrm{~ms}$; (c) burst duration $=50 \mathrm{~ms}$ ].

ments. This criterion was not exceeded at a burst duration of $10 \mathrm{~ms}$, and the ISOI range at higher burst durations was not affected by the arrangement of the speakers.

Figure 8 presents the percentage of correct direction reports for each speaker arrangement. Performance on this task was affected by the arrangement of the speakers and was best in the horizontal condition. The difference in performance between the vertical and front-back dimensions is smaller, and performance was better in the front-back dimension. Significant main effects of speaker arrangement $[F(2,8)$ $=20.07 ; p<0.0008]$, burst duration $[F(2,8)=5.4$; $p<0.03]$, and ISOI $[F(9,36)=17.76 ; p<0.0001]$ were found. Significant interactions of speaker arrangement by ISOI $[F(18,82)=2.09 ; p<0.01]$ and duration by ISOI $[F(18,72)=2.68 ; p<0.002]$ were also obtained. In this analysis however, the main effect of speaker arrangement accounted for $23 \%$ of the total variance. Adopting a $75 \%$ detection criterion, a total duration of roughly $75 \mathrm{~ms}$ is required for the identification in the front-back dimension. In the vertical dimension, the $75 \%$ criterion was not reached.

The results of experiment 3 essentially replicate those of experiment 1 . The absence of binaural localization cues did not affect the timing values that produced AAM. These cues only affected whether the direction of motion was detected.
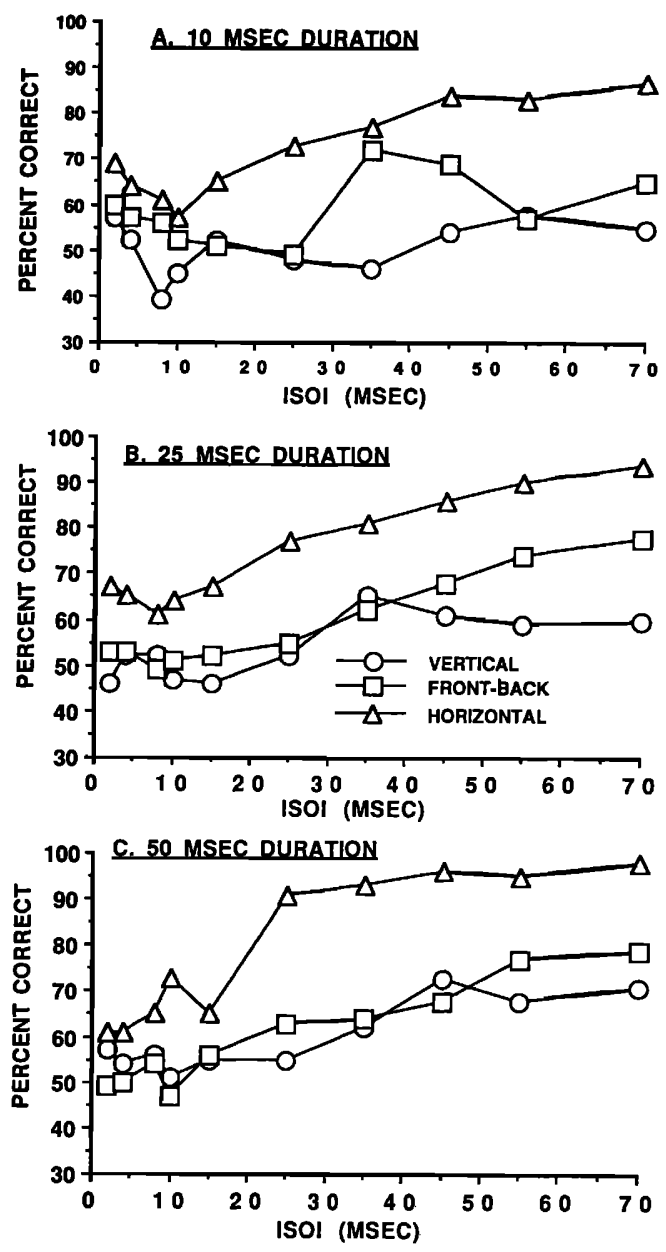

FIG. 8. The mean percentage of correct judgments the direction of the lead source as a function of ISOI and the arrangement of the speakers [(a) burst duration=10 ms; (b) burst duration=25 ms; (c) burst duration $=50 \mathrm{~ms}$.

In the present experiment, the spectrum of the stimuli were more likely to produce monaural shape cues required for localization in the median plane. When the speakers were arranged vertically or in the front-back dimension, continuous motion was heard at the same combinations of duration and ISOI that produce the perception of continuous motion in the horizontal plane. ${ }^{6}$

\section{EXPERIMENT 4: THE EFFECT OF SPEAKER SEPARATION ON AAM IN THE VERTICAL PLANE}

In each of the experiments reported here, the location of the speakers had little or no effect on the perception of AAM. Neither the elimination of interaural difference cues in experiment 1, variation in the number of sources used in experiment 2 , nor the plane in which the sources were separated affected the conditions that produced AAM. All of these manipulations did affect detection of direction of motion, however. Taken together, these results could be explained by assuming that static location cues are not required for the perception of motion in the auditory modality, but are necessary for detecting the direction of movement. On the basis of this assumption we would predict that AAM could be heard when the separation between the sources is less than the MAA. 
Another interpretation that is consistent with the results of experiments $1-3$ is that the obtained continuous motion distributions were caused by some experimental artifact. In each experiment reported here, burst duration was not varied within a block of trials, and the same set of ISOIs were tested across several trial blocks. Our subjects may have simply learned to respond with the continuous motion category at some ISOI range. It is also possible that the set of response categories used was inappropriate for the manipulations of experiments 1-3, and forced subjects to respond with the motion categories even when no motion was heard. For example, if at some ISOI the stimulus sequence was clearly not single, simultaneous or successive, the only choices left were motion categories (continuous or broken).

The final experiment tested both interpretations of the results of experiments 1-3. It was determined if AAM could be heard when the sound sources were separated by less than one MAA, and if contextual cues could account for the results. AAM was measured in the vertical plane at two separations, $2.5^{\circ}$ and $20^{\circ}$. A separation of $2.5^{\circ}$ is less than the vertical MAA reported by Perrott and Saberi (1990). The $20^{\circ}$ separation is a replication of the vertical condition of experiment 3. Some subjects were tested with two ranges of ISOIs. Others were tested when the burst duration was varied within trial block and an alternative set of response categories were used. This new set was created by substituting "none of the above" for the simultaneity category used previously.

\section{A. Method}

\section{Subjects}

Four subjects were tested. Two of these had participated in experiments 2 and 3 . The other two had previous experience with AAM, but did not participate in experiments 1-3. One of these had hearing within normal limits and the other (TZS, one of the authors) had some hearing loss at frequencies above $5 \mathrm{kHz}$. All subjects were tested at vertical separations of $2.5^{\circ}$ and $20^{\circ}$. Two subjects were tested for the effects of ISOI range on AAM, and three were tested for the effects of burst duration and response set (one subject was used in both tests).

\section{Apparatus}

The apparatus was the same as in experiment 3 with the following modifications. All of the surfaces of the audiometric chamber were covered with Sonex 4 in acoustical sheets. These sheets report absorption coefficients of at least 1.06 for frequencies above $0.25 \mathrm{kHz}$. Three speakers were used in this experiment. One was positioned at $0^{\circ}$ azimuth and $0^{\circ}$ elevation relative to the subject. The other speakers were positioned so as to produce vertical separations of $2.5^{\circ}$ and $20^{\circ}$, each at $0^{\circ}$ azimuth. The subject's chair was also moved to produce the desired vertical separation. For the $2.5^{\circ}$ separation, the speakers were $2.7 \mathrm{~m}$ from the subject, and for $20^{\circ}$ the speakers were $1.5 \mathrm{~m}$ from the subject. The stimulus consisted of high-pass noise $(2-16 \mathrm{kHz})$ at $50 \mathrm{~dB}$ A-weighted.

\section{Procedure}

Two separations $\left(2.5^{\circ}\right.$ and $\left.20^{\circ}\right)$ and two ISOI ranges were used in the test of ISOI range. The short ISOI range consisted of $2,4,8,10,15,25,35,45,55$, and $70 \mathrm{~ms}$, and the long ISOI range consisted of $10,20,30,50,70,90,130,170$, 210 , and $250 \mathrm{~ms}$. This created a set of four trial blocks, presented in random order. Burst duration was always $50 \mathrm{~ms}$. Within a trial block, each ISOI was presented 30 times in random order. Half of these originated at $0^{\circ}$ elevation, and half at the higher elevation. Subjects were instructed to classify their perceptions into one of the five categories used in experiments 1-3, and to indicate the location of the lead source (down or up).

In the test of response set, two durations (50 and 100 ms) and 10 ISOIs $(1,10,20,30,40,50,60,70,80,90$, and $100 \mathrm{~ms}$ ) were presented 12 times each within a trial block. Two blocks of trials were run at each separation, providing a total of 24 trials at each separation-duration-ISOI combination. In addition, two sets of response categories were tested, making a total of eight trial blocks. The first response category set was the same as in experiments $1-3$. The second set substituted "none of the above" for the simultaneous category. Simultaneous was omitted because it was rarely used when AAM was produced in the vertical plane in experiment 3 . The none-of-the-above category was defined as a perception that could not be classified into any of the other categories. Subjects were again instructed to indicate the location of the lead source (up or down). However, feedback was now provided to the subject on the direction task.

\section{B. Results and discussion}

Figure 9(a) presents the mean percentage of continuous motion responses as a function of vertical separation and ISOI range. The continuous motion distributions are similar at both separations and ISOI ranges. Figure 9(b) presents the results of the direction task. Performance is clearly superior at $20^{\circ}$ separation, although the mean percent correct curve never reached a $75 \%$ correct criterion at any ISOI. ${ }^{7}$

Figure 10 presents the percentage of continuous motion responses as a function of duration and response set, and Table I presents the upper and lower thresholds for continuous motion. At a separation of $2.5^{\circ}$, shown in Fig. 10(a), continuous motion was reported at ISOIs greater than $20 \mathrm{~ms}$ for the 100-ms burst duration, and at ISOIs of 33-52 ms for a burst duration of $50 \mathrm{~ms}$. However, at the 50-ms duration, less continuous motion is reported overall. In Fig. 10(b), continuous motion is heard at both durations at roughly the same range of ISOIs for the corresponding burst duration in Fig. 10(a). The peaks of the 50-ms distributions are of the same magnitude as the peaks of the 100 -ms conditions. In Fig. 10(a) and (b), there are no differences between response sets on the percentage of continuous motion. The none-ofthe-above category of response set 2 was rarely used. The mean percentage none-of-the-above reports reached a maximum of $18 \%$ at a separation of $20^{\circ}$, burst duration of $50 \mathrm{~ms}$ and ISOI of $20 \mathrm{~ms}$.

Figure 11 presents the results of the direction task. Performance is at chance levels at all ISOIs and durations when 

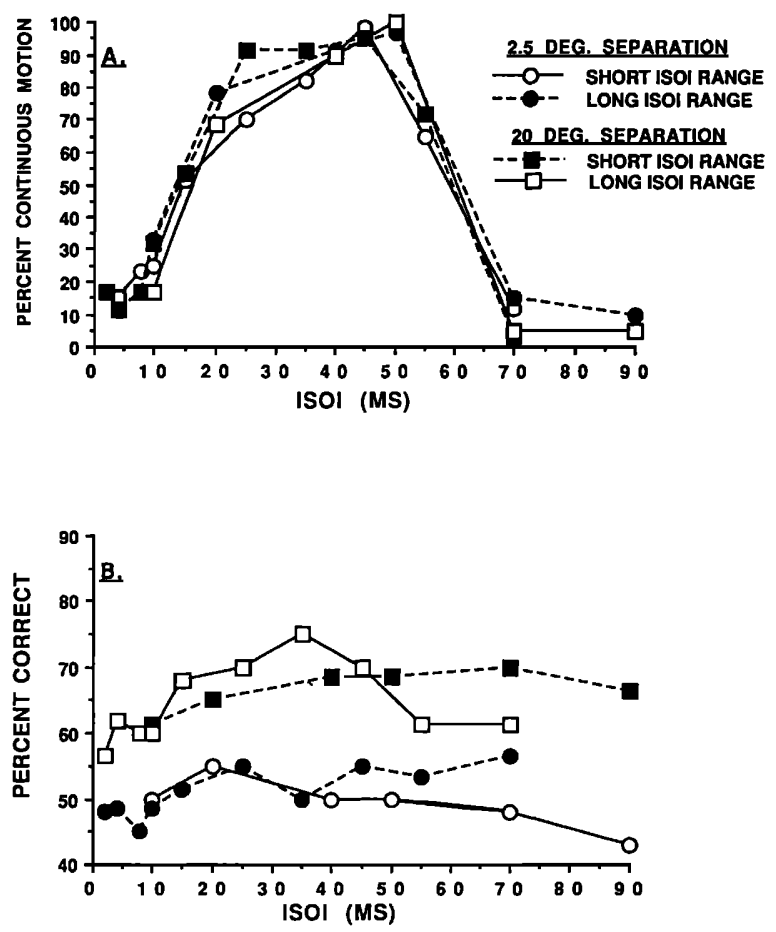

FIG. 9. The mean percentage of (a) continuous motion responses and (b) correct direction judgments as a function of ISOI for vertical separations of $2.5^{\circ}$ and $20^{\circ}$.

the separation is $2.5^{\circ}$. At $20^{\circ}$, subjects reliably discriminated between the sources for ISOIs exceeding $25-30 \mathrm{~ms}$. Feedback improved performance over that obtained in the first phase of this experiment and in the vertical condition of experiment 3 only when the separation between the sources exceeded the vertical MAA. ${ }^{8}$

These results appear to rule out the contextual factors of ISOI range, number of burst durations tested within a trial block and response set as potential explanations for the perception of AAM. Furthermore, the results suggest that a detection of a difference in the location of the two sources is not a necessary requirement for the perception of AAM. However, when the two sources are discriminable in location, more motion is reported at the shorter durations.

\section{GENERAL DISCUSSION}

One finding that was observed in each of these experiments on AAM is that the perception of motion does not require that the location of individual sources be detected. Cues to source location are critical only for the detection of the direction of motion, not for the detection of motion per se. Temporal parameters alone determine whether AAM is heard. Both spatial and temporal parameters determine whether the direction of motion can be identified. These conclusions are consistent with previous research on AAM.

AAM is determined by the appropriate timing values of burst duration and ISOI only. AAM was heard at the same combinations of burst duration and ISOI under binaural and monaural listening conditions, with two and three sound sources, with sources separated in the horizontal and median planes and when the separation was less than the MAA. In
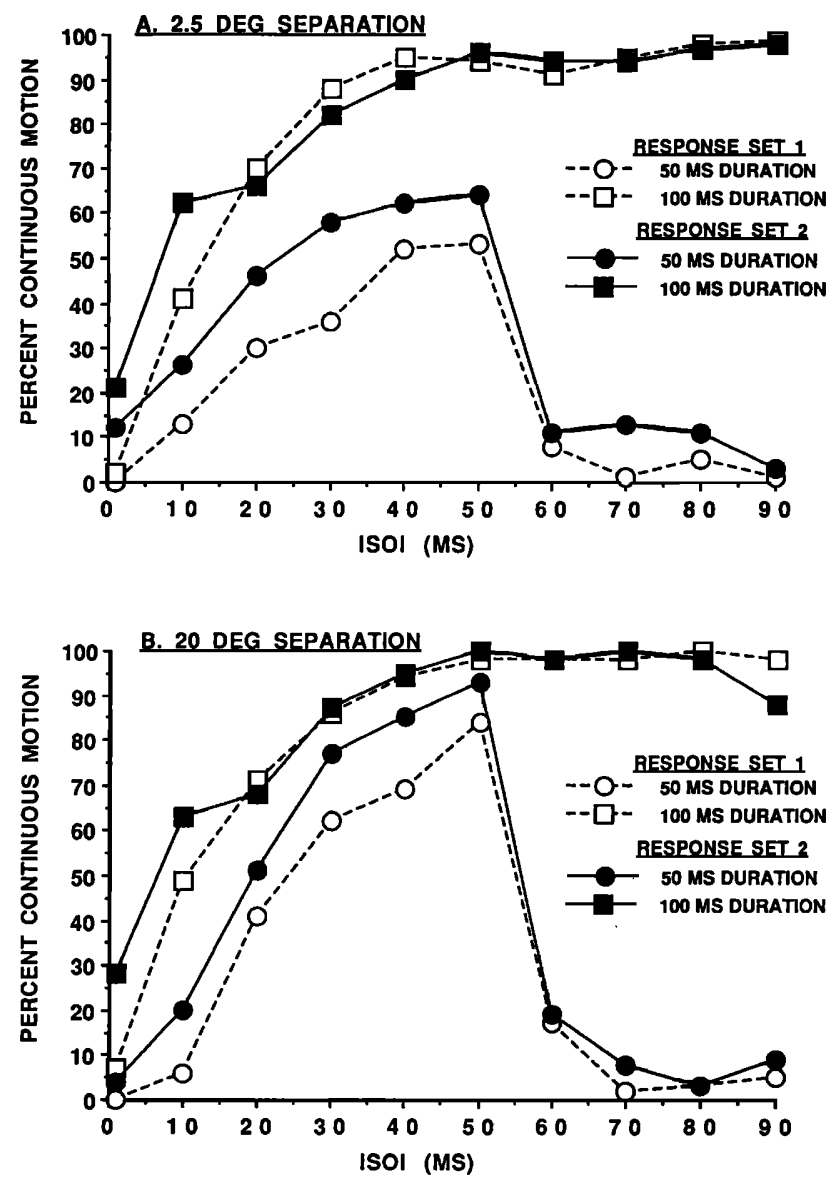

FIG. 10. The mean percentage of continuous motion responses as a function of ISOI, burst duration and response set $\left[(\mathrm{a})\right.$ vertical separation $2.5^{\circ}$; (b) vertical separation $20^{\circ}$.

previous research it has been shown that AAM is heard at roughly the same values of duration and ISOI under dichotic listening conditions (Briggs and Perrott, 1972) for separations between $2.5^{\circ}$ and $160^{\circ}$ for binaural listening (Strybel et al., 1990; Strybel et al., 1992) and between $10^{\circ}$ and $80^{\circ}$ for monaural listening (Strybel et al., 1989).

The results of these experiments do not completely agree with the conditions for continuous motion established previously by Strybel et al. (1992), however. These condi-

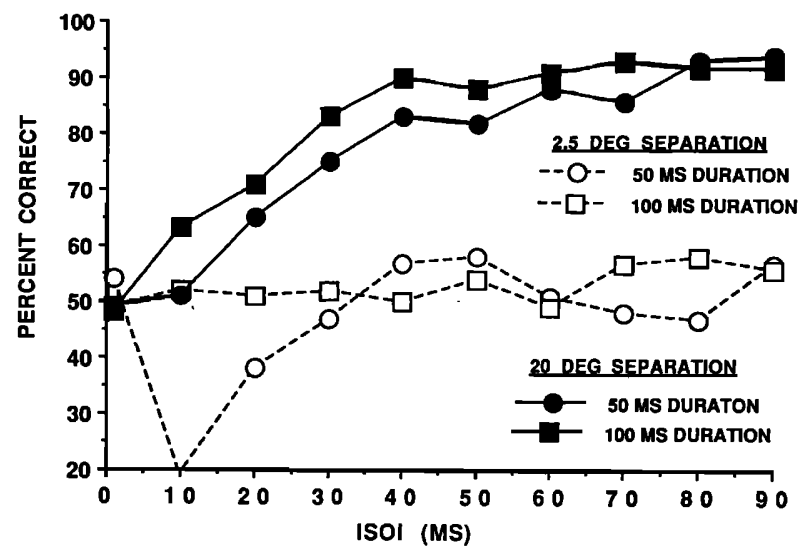

FIG. 11. The mean percentage of correct direction judgements as a function of ISOI, burst duration and vertical separation. 
tions were a minimum total duration of at least $100 \mathrm{~ms}$, and no silent gap. In experiment 1 , these rules were applicable only when the duration of each stimulus was less than 300 ms. At a burst duration of $300 \mathrm{~ms}$, ISOIs between 200 and $300 \mathrm{~ms}$ produced the perception of broken motion, even though these combinations meet the criteria of Strybel et al. Another difficulty with these requirements is that in all previous investigations of $\mathrm{AAM}$, total duration was confounded with burst duration and ISOI. In experiment 2 , when two and three sources were used, total duration could not reliably predict when AAM was heard. In this experiment, the burst duration and ISOI of contiguous events were the best predictors of the illusion. We can summarize the conditions for continuous motion as follows. The ISOIs that produce continuous motion are a function of burst duration. As the burst duration increases, the ISOIs that produce motion also increase. Moreover, the range of ISOIs that produce continuous motion increases with burst duration up to $100 \mathrm{~ms}$ (see Table I).

Determination of the direction of AAM on the other hand, depends on both temporal parameters and the availability of cues to the location of the individual sound sources. In experiment 1 , our subjects were unable to determine the direction of motion in the monaural listening condition at any burst duration-ISOI combination. In experiment 2 , direction of motion could be detected when the total duration of the stimulus sequence exceeded roughly $50 \mathrm{~ms}$, for both two and three sources. In experiment 3 , front-back direction of motion discrimination was possible at ISOIs greater than $55 \mathrm{~ms}$ at burst durations of 25 and $50 \mathrm{~ms}$, suggesting that total duration does not describe performance in the median plane. These minimum values may be improved with feedback, however, as in experiment 4 our listeners could discriminate the up-down direction at ISOIs in excess of $25 \mathrm{~ms}$ for burst durations of 50 and $100 \mathrm{~ms}$, and only when the separation exceeded the MAA.

What cue could listeners use to detect motion that is not dependent on static location information? One possible cue of this type is amplitude modulation. A sound moving around the head at a constant distance from the listener would produce changes in the amplitude of the signal arriving at each ear. Although the interaural differences in intensity could signal changes in source location, the monaural change in intensity over time is proportional to source velocity and could be used as a cue to movement (Zakaruskas and Cynader, 1991). In AAM experiments, motion is heard when two sound events either overlap or follow closely in time, producing a proximal stimulus which increases in amplitude. Under monaural listening conditions, the appropriately timed AAM stimulus would produce a monaural change in amplitude to the unoccluded ear. When the separation between the sources is less than one MAA, the AAM stimulus would produce an amplitude increase to both ears. The amplitude change produced in both of these conditions could signal movement and be ambiguous about direction of movement. A monaural motion detection mechanism of this type is consistent with research on other topics related to auditory motion perception, such as auditory motion aftereffects (e.g.,
Reinhardt-Rutland, 1992) and single-cell recording work on auditory motion detectors (e.g., Stumpf et al., 1992).

A mechanism for detecting amplitude modulation does not rule out a system that detects changes in location over time (the snapshot mechanism), however. In all of the experiments reported here, more motion was reported overall when the direction of movement was correctly identified. For example, in the monaural listening condition of experiment 1 , where location cues were degraded, less continuous motion was reported overall compared to the binaural condition. In experiment 4 , at the $2.5^{\circ}$ separation, less motion was reported at $50 \mathrm{~ms}$ relative to the $20^{\circ}$ separation. Thus a stimulus that provides information about movement in the form of amplitude modulation cues and changes in position is more likely to be reported as moving than one that provides only one cue to movement.

Although the possibility of a monaural motion detection mechanism is speculative at this time, the experiments reported here showed that the factors that produce the perception of motion are not the same as those that allow detection of direction. We have determined that the perception of AAM depends on burst duration and ISOI only, while the perception of the direction of AAM depends on the total duration of the stimulus sequence and the strength of static localization information.

\section{ACKNOWLEDGMENTS}

We would like to thank John Whittaker for his help in developing the apparatus used in experiments $2-4$ of this manuscript. The production of this publication was partially funded by a California State University Long Beach MiniGrant Award.

${ }^{1}$ The percentage of broken motion responses were also analyzed. In general, broken motion responses were made for ISOIs greater than $100 \mathrm{~ms}$. The relationship between the location of the peaks of these distributions and burst duration is similar to that obtained with continuous motion.

${ }^{2}$ Measurements of AAM, as well as MAMAs, are usually highly variable (e.g., Strybel et al., 1989; Grantham, 1986). The results obtained here were no exception, yet the trends observed with the mean percentages can also be seen with the individual data. For example, four out of six subjects reported continuous motion at least $50 \%$ of the time, at some range of ISOIs and at all durations and for both monaural and binaural listening conditions. One of the remaining subjects (VP) did not report continuous motion at the 10 -ms duration for monaural or binaural listening. The sixth subject $(\mathrm{AL})$ rarely reported continuous motion at any duration and listening condition. Only one subject (TS) ever exceeded a $75 \%$ correct detection of lead source location in the monaural listening condition. This performance was achieved at 50-ms duration for ISOIs between 70 and $150 \mathrm{~ms}$. Five of the subjects exceeded the $75 \%$ criterion in the binaural listening conditions at all durations tested.

${ }^{3}$ Broken motion was heard more than $70 \%$ of the time at burst durations of 5 and $10 \mathrm{~ms}$ and ISOIs greater than $15-\mathrm{ms}$ durations in the three source condition. When two sources were used to produce broken motion, it was heard for ISOIs in excess of $25 \mathrm{~ms}$ at 5 - and 10 -ms durations, and the magnitude of the peaks of these functions is considerably lower than in the three source condition.

${ }^{4} \mathrm{An}$ analysis of the individual subject data confirmed the conclusions reached with the mean data presented in the body of the paper. At the 5-ms duration, none of our subjects exceeded a $50 \%$ continuous motion report criterion in the two-speaker condition, and only one exceed this criterion in the three-speaker condition. All of our subjects reported continuous motion more than $50 \%$ of the time at durations of 25 and $50 \mathrm{~ms}$, for both the twoand three-speaker conditions, with little difference noticed between the ISOI range. For the percentage of correct direction judgments, the mini- 
mum ISOI that exceeded a $75 \%$ correct detection criterion for five subjects decreased with increases in burst duration. At each burst duration the minimum ISOIs were generally lower for the three-speaker condition compared to the two-speaker condition.

${ }^{5}$ The percentage of broken motion responses were also analyzed. The mean percentage of broken motion responses were higher in the horizontal arrangement of speakers, although the magnitude of the main effect of speaker arrangement was quite small (less than $1 \%$ of the variance). For all speaker arrangements, the minimum ISOI where broken motion was heard increased with burst duration.

${ }^{6} \mathrm{As}$ in experiments 1 and 2, the individual data were consistent with the mean data. At the 10-ms duration, two subjects reported continuous motion more than $50 \%$ of the time for the front-back and vertical speaker arrangements. Only one subject exceeded this criterion in the horizontal condition. At $50 \mathrm{~ms}$, four subjects exceeded the $50 \%$ criterion for the front-back and vertical conditions, and five subjects exceeded the criterion in the horizontal condition. The individual data on the percentage of correct location detection were also consistent with the mean data. In the vertical arrangement, none of our subjects reached a $75 \%$ correct detection level at the 10 -ms duration, one subject reached this level at the 25 -ms duration and two subjects at the 50-ms duration. In the front-back arrangement, two of our subjects exceeded the $75 \%$ correct level at the 10 -ms duration, three subjects at the $25-\mathrm{ms}$ duration and three subjects at the $50-\mathrm{ms}$ duration. In the horizontal condition four subjects exceeded this criterion at $10 \mathrm{~ms}$, and all five exceeded the criterion at higher durations.

${ }^{7}$ No difference was obtained between the two subjects on the percentage of motion reports. However, at $20^{\circ}$ separation subject JB exceeded the $75 \%$ criterion at an ISOI of $10 \mathrm{~ms}$, while the other subject (AW) never reached $75 \%$ correct at any ISOI.

${ }^{8}$ When feedback was provided all subjects exceeded $75 \%$ correct on the direction task with a minimum ISOI between 10 and $40 \mathrm{~ms}$. One of the subjects in this task was AW who did not reach $75 \%$ correct in the absence of feedback.

Altman, J. A., and Viskov, O. V. (1977). "Discrimination of perceived movement velocity for fused auditory image in dichotic simulation," J. Acoust. Soc. Am. 61, 816-819.

Braddick, O. J. (1974). "A short-range process in apparent motion," Vision Res. 14, 519-528.

Briggs, R., and Perrott, D. R. (1972). "Auditory apparent movement under dichotic listening conditions," J. Exp. Psychol. 92, 83-91.

Burtt, H. E. (1917). "Auditory illusions of movement-a preliminary study," J. Exp. Psychol. 2, 63-75.

Chandler, D. W., and Grantham, W. D. (1992). "Minimum audible movement angle in the horizontal plane as a function of stimulus frequency and bandwidth, source azimuth, and velocity," J. Acoust. Soc. Am. 91, 16241636.

Grantham, D. W. (1986). "Detection and discrimination of simulated motion of auditory targets in the horizontal plane," J. Acoust. Soc. Am. 79, 19391949.

Gregory, R. L. (1972). Eye and Brain: The Psychology of Seeing (McGrawHill, New York).
Kirk, R. E. (1968). Experimental design: Procedures for the Behavioral Sciences (Brooks/Cole, California).

Kolers, P. A. (1972). Aspects of Motion Perception (Pergamon, Oxford).

Mack, A. (1986). "Perceptual aspects of motion in the frontal plane," in Handbook of Perception and Human Performance Vol. 1. Sensory Processes and Perception, edited by K. R. Boff, L. Kaufman, and J. P. Thomas (Wiley, New York).

Middlebrooks, J. C., and Green, D. M. (1991). "Sound localization by human listeners," Ann. Rev. Psychol. 42, 135-159.

Mills, A. W. (1958). "On the minimum audible angle," J. Acoust. Soc. Am. 30, 237-246.

Newsome, W. T., Mikami, A., and Wurtz, R. H. (1986). "Motion selectivity in macaque visual cortex. III. Psychophysics and physiology of apparent motion," J. Neurophysiol. 55, 1340-1351.

Perrott, D. R., and Musicant, A. D. (1977). "Minimum audible movement angle: Binaural localization of moving sound sources," J. Acoust. Soc. Am. 62, 1463-1466.

Perrott, D. R., and Tucker, J. (1988). "Minimum audible movement angle as a function of signal frequency and velocity of the source," J. Acoust. Soc. Am. 83, 1522-1527.

Perrott, D. R., and Pacheco, S. (1989). "Minimum audible angle thresholds for broadband noise as a function of the delay between the onset of the lead and lag signals,"J. Acoust. Soc. Am. 85, 2669-2672.

Perrott, D. R., and Saberi, K. (1990). "Minimum audible angle thresholds for sources varying in both elevation and azimuth," J. Acoust. Soc. Am. 87, 1728-1731.

Reinhardt-Rutland, A. H. (1992). "Changing-loudness aftereffect following simulated movement: Implications for channel hypothesis concerning sound level change and movement," J. Gen. Psychol. 119, 113-121.

Saberi, K., and Perrott, D. R. (1990). "Minimum audible movement angles as a function of sound source trajectory," J. Acoust. Soc. Am. 88, 26392644.

Strybel, T. Z., Manligas, C. L., and Perrott, D. R. (1989). “Auditory apparent motion under binaural and monaural listening conditions," Percept. Psychophys. 45, 371-77.

Strybel, T. Z., Manligas, C. L., and Perrott, D. R. (1992). "Minimum audible movement angle as a function of the azimuth and elevation of the source," Human Factors 34, 267-75.

Strybel, T. Z., Manligas, C. L., Chan, O., and Perrott, D. R. (1990). "A comparison of the effects of spatial separation on apparent motion in the auditory and visual modalities," Percept. Psychophys. 47, 439-448.

Strybel, T. Z., Witty, A. M., and Perrott, D. R. (1992). "Auditory apparent motion in the free field: The effects of stimulus duration and separation," Percept. Psychophys. 52, 139-143.

Stumpf, E., Toronchuk, J., and Cynader, M. (1992). "Neurons in the cat primary auditory cortex sensitive to correlates of auditory motion in threedimensional space," Exp. Brain Res. 88, 158-168.

Viskov, O. V. (1975). "The perception of the fused auditory image movement," Human Physiol. 1, 371-376.

Zakaruskas, P., and Cynader, M. S. (1991). "Aural intensity for a moving source," Hear. Res. 52, 233-244. 